\title{
Functional Outcome of Fixation of Complex Intra-articular Distal Radius Fractures with a Variable-Angle Distal Radius Volar Rim Plate
}

\author{
$\begin{array}{llll}\text { M. Spiteri } & \text { W. } \mathrm{Ng}^{1} & \text { J. Matthews } & \\ & & \text { D. Power }\end{array}$ \\ ${ }^{1}$ Hand Unit, Queen Elizabeth Hospital, Birmingham, United Kingdom \\ J Hand Microsurg 2017;9:11-16.
}

Address for correspondence M. Spiteri, MD, MRCS, MSC, FRCS, FEBOT, Hand Unit, Nuffield Orthopaedic Centre, Oxford University Hospitals, Oxford OX3 9HE, United Kingdom

(e-mail: michespi@gmail.com).

\begin{abstract}
Keywords

- distal radius fracture

- open reduction and internal fixation

- patient evaluation measure

- variable-angle distal radius rim plate

Aim To evaluate the outcome of these complex fractures using a volar approach and the DePuy Synthes variable-angle 2.4-mm distal radius rim plate. This plate is precontoured to the volar rim for placement distal to the watershed line allowing purchase of the rim fragment of the lunate facet. Its low profile and smooth edges are designed to minimize flexor tendon irritation.

Method We report on a consecutive series of far distal AO-23B3 and AO-23C3 fractures treated using this plate in a tertiary hand center between November 2011 and May 2014. Range of motion, grip strength, and complications were assessed at the final clinical review. Disabilities of the arm, shoulder, and hand (DASH) and patient evaluation measure (PEM) scores were assessed at 12 months after surgery.

Results Twenty-six patients were included in this review. Six patients were lost to follow-up at 3 months. This plate was used in isolation in 17 cases, and in combination with a dorsal plate, in cases of dorsal instability after volar plating, in 10 patients. DASH and PEM scores 1 year after surgery were 17.6 and 27\%, respectively. Visual analog scores for patient treatment satisfaction and severity of pain showed good satisfaction with treatment and mild intermittent pain on activity. Postoperative range of motion was variable and grip strength was of $71 \%$ of the uninjured contralateral side. There were no cases of flexor or extensor tendon rupture. Tendon irritation was noted in two patients. Removal of metal was performed in four patients. Loss of reduction occurred in one case and neurologic complications in two cases.

Conclusion This implant is specifically designed for the management of far distal complex intra-articular fractures of the distal radius. Despite the complexity of these fracture patterns and the challenge they pose to accurate reduction and stable fixation, outcomes were satisfactory in this small series. There were no cases of tendon rupture. Removal of metal is not necessary in all cases, but prompt removal should be performed in cases of tendon irritation in view of the risk of tendon rupture.
\end{abstract}

\section{Introduction}

Marginal intra-articular distal radius fractures pose a significant challenge to the treating surgeon. The fracture line is at

received

December 3, 2016

accepted after revision

February 17, 2017

published online

March 24, 2017

or distal to the watershed line ${ }^{1}$ and is not always buttressed by standard volar distal radius plate placement at or proximal to the watershed line. This can lead to secondary volar displacement of the volar lip fragment with subluxation of

(c) 2017 Society of Indian Hand \& Microsurgeons
DOI http://dx.doi.org/ 10.1055/s-0037-1601325. ISSN 0974-3227. 
the carpus. In the distal AO-23C3 subtype successful buttressing for volar instability may also unmask dorsal instability that might require supplementary dorsal plate fixation.

The challenge in treating these far distal fractures has resulted in a variety of management options, including spanning external fixation, bridge plating techniques, and tension band or fragment-specific fixation, ${ }^{2,3}$ The latter can be performed by open reduction and internal fixation (ORIF) using solitary volar or dorsal approaches or a combination of both, and may be arthroscopically assisted.

The various volar locking plate designs available in recent years enable reliable fixation of most fracture subtypes, including certain dorsally unstable distal radius fractures. These offer the advantage of better soft tissue cover and less extensor tendon compromise when compared with a dorsal approach. Fracture stability through a full range of wrist movement should be assessed intraoperatively after plating, especially in the fractures that are distal to the watershed line, and addressed with an additional approach if necessary.

If a standard volar plate designed for placement proximal to the watershed line is used in these far distal fractures, the fracture will require additional fixation distal to the plate with lag screws or suture wires to secure the marginal lip fracture. Attempts at capturing the dorsal fragments with distally directed variable-angle screws through the plate may increase the risk of screw penetration of the joint.

This variable-angle distal radius rim plate was designed to provide more reliable fixation of these uncommon far distal complex intra-articular fractures. Its design is precontoured to the volar rim to allow placement distal to the watershed line and purchase or buttress of the volar rim of the lunate facet buttress. The plate was introduced to our tertiary referral hand unit in 2011 for the selective treatment of these fracture subtypes.

This retrospective study reports on the results of a consecutive series of far distal AO-23B3 and AO-23C3 fractures treated with the DePuy Synthes variable-angle 2.4-mm distal radius rim plate (Oberdorf, Switzerland) at a major trauma and tertiary referral hand center between November 2011 and May 2014.

\section{Methods}

Fracture configuration was classified radiographically by a hand surgeon. Further assessment of fragment comminution and displacement was obtained by means of computed tomography scans in all cases.

Patients were identified using a database of distal radius fractures compiled from theater logbooks and cross-referenced with implant logbooks. Medical and therapy records were reviewed to evaluate complications and assess range of motion measured with a goniometer and grip strength using a Jamar dynamometer with the elbow unsupported in 90 degrees of flexion and elbows tucked by the side. Grip strength was compared with the uninjured contralateral limb and expressed as a percentage of this.

The primary outcome measures were range of motion and grip strength at final clinical review at 1 year. Secondary outcome measures were the need for further surgery and complications. Functional outcome measures used included the disabilities of the arm, shoulder, and hand (DASH) score and the patient evaluation measure (PEM). These upper limb scores have similar validity and reliability. The PEM score is also the only outcome measure validated in terms of content regarding distal radial fractures, as it was specifically evaluated for such injuries. ${ }^{4}$ They were given to patients in clinic at 12 months after surgery. This was returned on the day or by post. In half the patients an attempt to quantify preinjury assessment of level of function was performed by means of a DASH score. In these cases, the patient was asked to complete the score on the day of surgery, prior to his/her operation.

\section{Results}

During the study period, 382 ORIF operations upon the distal radius were performed in our institution. Twenty-seven (6.8\%) patients had fracture fixation using this implant. The plate was used in isolation in 17 cases, and in combination with an additional dorsal plate, in a buttress mode, in 10 (35\%) patients to control dorsal fragment instability, achieve better reduction and stability of the dorsoulnar fracture fragment, and achieve reconstruction of the sigmoid notch. Fracture stability was assessed using fluoroscopy intraoperatively through a full range of wrist movement after volar plating.

One patient was excluded from the study as he had a concomitant scapholunate ligament disruption requiring repair at the time of fixation of the distal radius fracture.

Patients were between 28 and 68 years of age, with a mean age of 40 years. Hypertension was the only comorbidity preoperatively. None of the cases were open fractures and none reported neurologic symptoms or signs prior to surgery.

All fractures treated with this plate had similar configurations (AO-23C3) with far distal articular comminution and die-punch involvement of the lunate fossa on CT scan (-Fig. 1). Simple distal radioulnar joint (DRUJ) coronal fracture lines were present in $20 \%$ of patients, and the remainder demonstrated DRUJ articular comminution on CT scans (-Fig. 2). The lunate facet had articular comminution involving the dorsoulnar and volar-ulnar regions of the distal radius as described by Melone. ${ }^{5}$ In the radial styloid fragment, comminution was mainly metaphyseal.

Fracture reduction was performed by means of ligamentotaxis, with intrafocal reduction in cases of central fragment depression. Alignment was restored to volar tilt of $4.3 \pm 5.5$ degrees, mean radial inclination of 21.2 degrees (range: $15-$ 30 degrees), $11.7 \mathrm{~mm}$ (range: $7-16 \mathrm{~mm}$ ) radial height, and $-1.2 \mathrm{~mm}$ (range: -4.5 to 2.5 ) ulnar variance. Postoperative loss of reduction occurred in one case, with loss of volar tilt and height. No intervention was required and the patient was asymptomatic at 18 months after surgery.

Six patients were lost to clinical follow-up: in two cases, patients declined review as they lived outside the region; in the other four cases, patients failed to attend clinic appointments beyond 12 weeks of surgery. All these six patients 

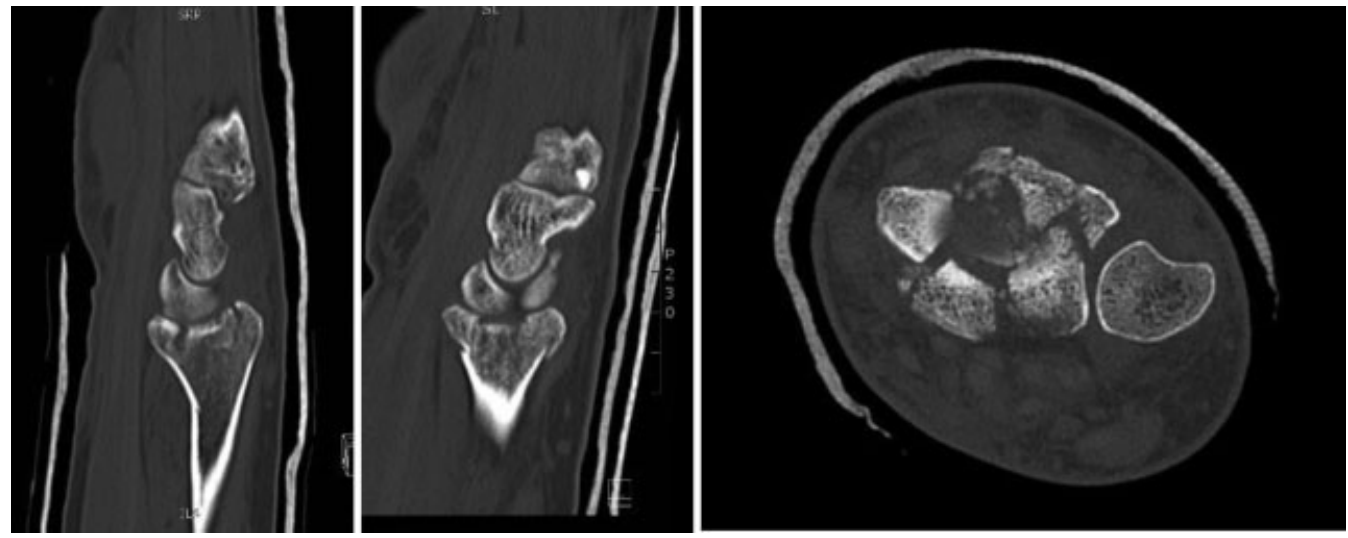

Fig. 1 Sagittal and axial computed tomographic scans showing involvement of lunate fossa and far distal articular comminution.

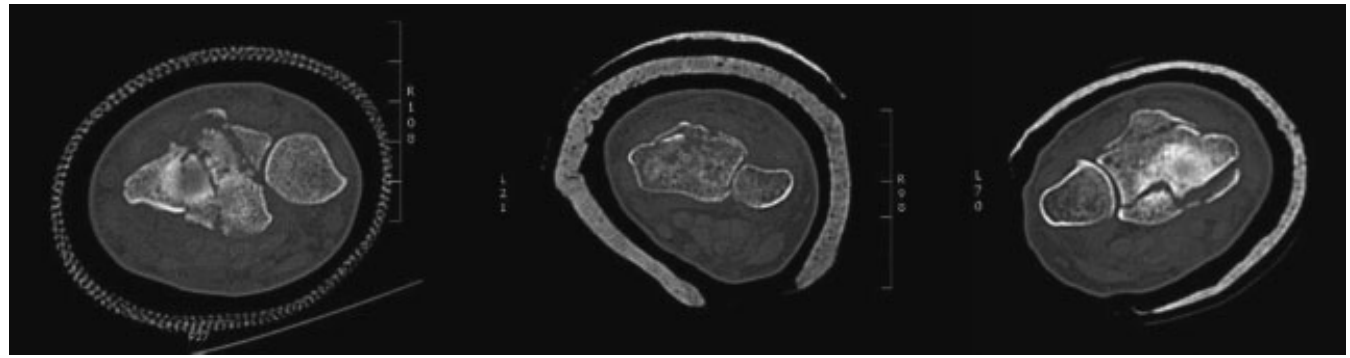

Fig. 2 Axial computed tomographic scan showing varying degrees of involvement of distal radioulnar joint.

were not referred back to our unit with complications. All 20 patients who attended follow-up reviews at our unit had radiographic union by 12 weeks following surgery.

All 26 patients completed the final functional outcome scores. This was sent in the post if patients did not attend clinical review (six cases mentioned previously). Clinical assessment including range of motion and grip strength was available for the 20 patients who completed follow-up (77\% of the total number of patients). The mean DASH and PEM scores at 12 months postoperatively were 17.6 (range: $3-74$ ) and $27 \%$, respectively.

The distribution of postoperative range of motion varied as shown in - Fig. 3, with a mean of 43 degrees of dorsiflexion (range: 5-80), 39 degrees of palmar flexion (range: 10-60), 18 degrees of radial deviation (range: 10-20), 19 degrees of ulnar deviation (range: 5-30), 65 degrees of pronation (range: 20-70), and 57 degrees of supination (range: $15-80$ ). Mean grip strength was $71 \%$ of the uninjured contralateral side.

Removal of implant was undertaken in $4(20 \%)$ of the 20 patients completing follow-up. There were no cases of flexor or extensor tendon rupture. Tendon irritation was noted clinically in two patients: one patient had first extensor compartment tendon irritation due to a prominent screw after fixation through an isolated volar approach; the other patient had flexor tendon irritation with crepitus and isolated volar sided pain on power grip. Fracture fixation in this case was done via a combination of volar and dorsal approaches. In both cases symptoms and clinical signs resolved on removal of the implants.

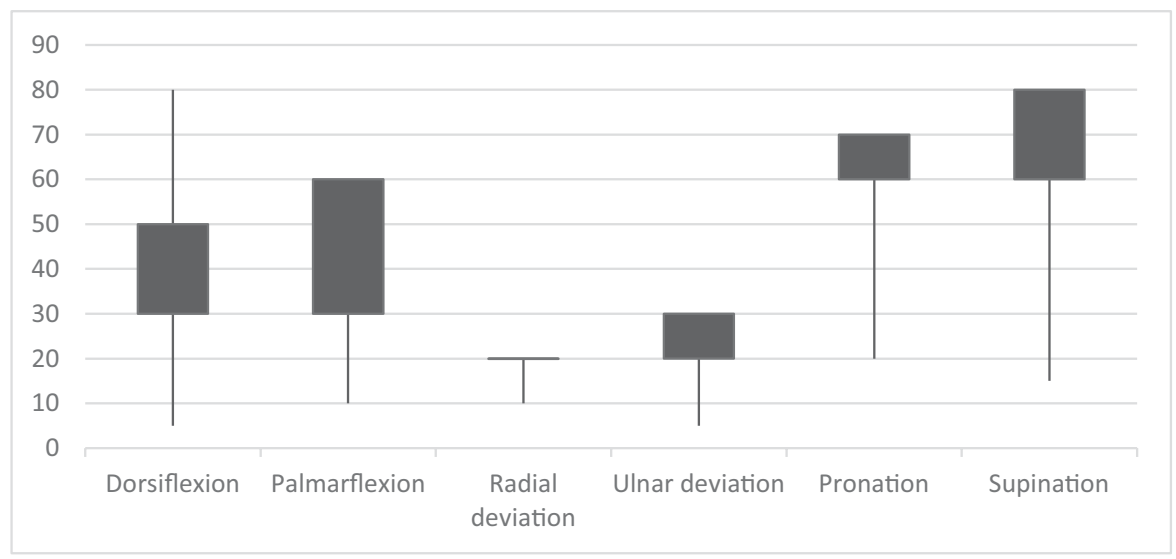

Fig. 3 Range of motion after open reduction and internal fixation. 
Implant removal was performed in a further two patients. This was due to new-onset dorsoradial pain after a fall 3 months after surgery. In this case screw penetration of the radiocarpal joint was identified arthroscopically at the time of removal of the isolated volar plate that had been used for fracture fixation. In the second case, the plate was removed at the time of secondary triangular fibrocartilaginous complex repair for symptoms of DRUJ instability.

On contacting the six patients who did not complete follow-up at our unit until 1 year after surgery, they denied symptoms of tendon irritation or rupture and did not have the implant removed.

The only other complications noted were neurologic, in two cases. One patient reported decreased sensation in the middle finger consistent with a Sunderland type II median nerve partial axonotmesis injury possibly related to retraction, which showed clinical signs of regeneration by 3 months following surgery. The second patient reported altered sensation in the distribution of the palmar cutaneous branch of the median nerve. This patient discontinued follow-up beyond 12 weeks of surgery; therefore, final outcome is not known.

\section{Discussion}

The operative management of distal radius fractures with ORIF has increased dramatically with the introduction of volar locking plates. Restoration of anatomy, joint congruity, and stability allows early functional mobilization; however, recent randomized controlled studies have questioned whether there are demonstrable functional advantages over more conservative methods of treatment in extraarticular and simple intra-articular fractures that may be reduced by closed manipulation. ${ }^{6,7}$ Comparative studies for complex articular comminuted fracture configurations are not available. This plate enables reliable reduction in far distal intra-articular $\mathrm{AO}-23 \mathrm{C} 3$ and $\mathrm{AO}-23 \mathrm{~B} 3$ fractures that may not otherwise be amenable to solitary fixation using a more traditional volar plate. In a study by Beck et al, fractures having marginal lunate fossa fragments with more than $5 \mathrm{~mm}$ subsidence will benefit from additional fixation distal to a standard plate in view of the risk of loss of reduction. ${ }^{8}$

Restoration of the anatomical parameters of the distal radius and DRUJ congruity were restored to within normal variations. Volar tilt was most difficult to restore due to both the complexity of the fracture, metaphyseal comminution, and far distal placement of the plate for adequate implant positioning and fixation.

The DASH score at 1 year was 17.6 , which is slightly higher than the outcomes of between 8 and 15 points quoted in the literature for AO-type $C$ fractures. ${ }^{1,9-13}$ This could be due to the increased complexity of this specific subset of type $C$ far distal fractures. There was a wide range of scores, 3 to 74 , which on further assessment reflects preinjury level of function. When the preinjury DASH scores were considered, these were increased, mean 3.3, in half the patients, which equates to a quarter of the total number of patients. Patients who had a higher DASH score preoperatively also had a higher postoperative score, demonstrating that preinjury level of function affects final outcome.

The PEM score is infrequently used to assess outcome of distal radius fractures despite its proven validity for these injuries. ${ }^{3}$ A score of $27 \%$ is reasonable considering the nature and complexity of the injury, as the score assesses not only pain and function but also patient satisfaction and perception. A range of 8 to $26 \%$ is described in the literature, but this takes into consideration all distal radius fractures treated with ORIF rather than this subset of far distal fractures. ${ }^{6}$ Pain severity and duration are assessed on a scale of 1 to 7 in this questionnaire, with 1 indicating no pain and 7 indicating unbearable pain. Patients scored a mean of 3 points. The majority also felt that the frequency of their pain was 2 points on the visual analog scores (VAS) of 1 to 7 , in which 1 is never and 7 is constant.

Overall patient satisfaction with treatment of their injury was rated at a mean of 2 points on the similar VAS in which 1 point is very satisfactory and 7 is very unsatisfactory.

The mean flexion and extension arc of 82 degrees and grip strength of $71 \%$ of the contralateral uninjured limb are similar to outcomes of AO-23C fractures described in the literature, even though this series includes only those with articular comminution and far distal fracture configuration. ${ }^{9,13,14}$ Forearm rotation, although limited at end range, is comparable to other studies for similar fractures. ${ }^{12}$

As the rim plate is designed to sit distal to the watershed line (-Fig. 4), tendon irritation and attritional rupture are potential risks when using this implant.

Flexor tendon irritation and rupture with the use of volar plates is well described in the literature. This is mainly due to high-profile implants sited at the watershed line, incorrect distal implant placement, or plate prominence due to failure of adequate restoration of volar tilt. ${ }^{15,16}$ Extensor tendon irritation and rupture generally occurs due to incorrect screw length or placement, which should be avoided and corrected intraoperatively. ${ }^{13}$ In this study, there were no cases of either flexor or extensor tendon rupture. Within the 1-year follow-up period, there was one case each of flexor

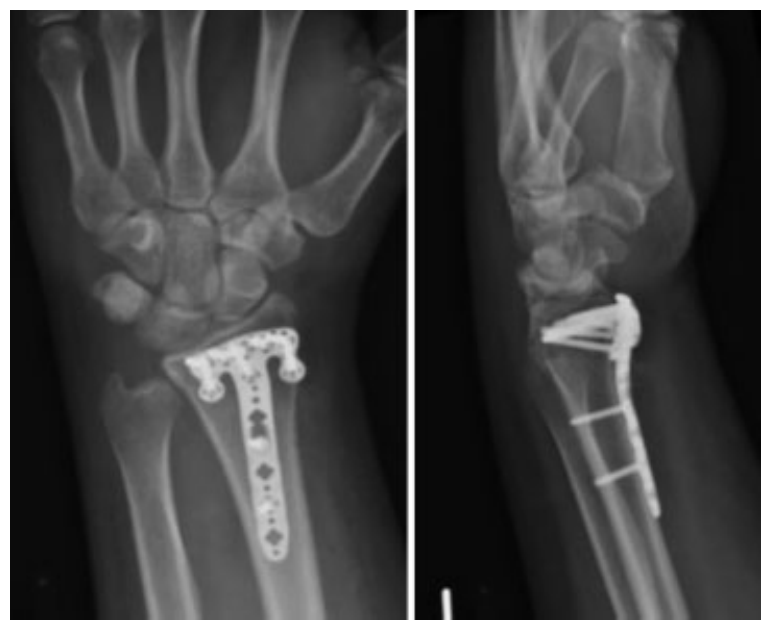

Fig. 4 PA and lateral radiographs of distal radius fracture showing distal position of rim plate. 
and extensor tendon irritation, which resolved on removal of the implants. Both patients became symptomatic and developed signs of tendon irritation when range of wrist motion had improved by 3 months of surgery. This would suggest that patients should be reviewed in clinic until a minimum of 6 months after surgery, when most of the wrist motion has been restored. We would recommend that if there are signs of tendon irritation at this stage, removal of the implants should be undertaken to avoid attrition ruptures.

Screw penetration into the radiocarpal joint occurred in one patient who developed new-onset pain after a second fall requiring removal of the implant. This patient had radiologic signs of collapse of the lunate fossa fragment compared with the postoperative radiographs. This made it difficult to establish whether screw penetration occurred intraoperatively or secondary to the second fall, due to displacement of the bone about the fixed screw. Even though the patient developed wrist pain after the second fall, it would imply delayed screw penetration.

Overall, removal of the plate was required in four cases. This is hard to compare with other studies and centers, as implants are sometimes removed routinely in view of the risk of tendon irritation.

Injuries to the median nerve and palmar cutaneous branch were the only other complications encountered in this series. None of the patients had preoperative median nerve symptoms at the time of injury or prior to surgery; hence, carpal tunnel decompression was not performed intraoperatively. These nerve injuries are described in the literature with varying results. In our series these did not seem to affect patient outcome scores as the symptoms improved with time. This is not always the case, and some patients can remain symptomatic with partial recovery, neuroma formation, or chronic neuropathic pain that is difficult to treat. ${ }^{13,17}$ Therefore, soft tissue care is of utmost importance when dealing with these complex injuries. The rate of nerve injuries associated with fixation of distal radial fractures using a volar approach is varied in the literature, ranging between 2 and $12 \%$ for nerve compression ${ }^{18,19}$ and 1.6 and $4.3 \%$ for pain syndromes. ${ }^{20,21}$ A recent meta-analysis describes this as a relative risk of nerve injury and neuropathy of 2.19 (95\% confidence intervals [CIs] 1.27, 3.76) and for postoperative nerve irritation with symptoms of carpal tunnel syndrome of 4.56 (95\% CIs $1.02,20.44){ }^{22}$

\section{Limitations}

This is a small case series; therefore, it was not possible to reliably compare the outcomes of solitary volar and combined volar and dorsal plating. A study assessing long-term follow-up of these patients should be considered in view of the risk of delayed flexor tendon rupture that has been reported with traditional more proximally placed volar plates.

\section{Conclusion}

This variable-angle distal radius volar rim plate is designed for the management of far distal complex intra-articular fractures of the distal radius. Despite the challenge these fractures pose to accurate reduction and stable fixation, the outcomes are satisfactory in this small case series. Removal of implant was performed in four cases. Complication rates were low, and there were no cases of flexor or extensor tendon rupture despite distal plate placement, but we recommend prompt removal of implant in cases of tendon irritation due to the higher morbidity associated with tendon rupture.

Note

There were no funding, research grants, or conflict of interest for any of the authors for this study.

This study does not involve research with human participants. The data and outcome measures used in the study were collected with the patients' informed consent as part of their routine follow-up clinic appointment after surgery to treat their injuries.

\section{References}

1 Jupiter JB, Marent-Huber M. Operative management of distal radial fractures with 2.4-millimeter locking plates. A multicentre prospective case series. J Bone Joint Surg Am 2010;92(1):96-106

2 Chen NC, Jupiter JB, Jupiter J. Management of distal radial fractures. J Bone Joint Surg Am 2007;89(9):2051-2062

3 O'Shaughnessy MA, Shin AY, Kakar S. Volar marginal rim fracture fixation with volar fragment-specific hook plate fixation. J Hand Surg Am 2015;40(8):1563-1570

4 Forward DP, Sithole JS, Davis TR. The internal consistency and validity of the patient evaluation measure for outcomes assessment in distal radius fractures. J Hand Surg Eur Vol 2007;32(3): 262-267

5 Melone CP Jr. Articular fractures of the distal radius. Orthop Clin North Am 1984;15(2):217-236

6 Karantana A, Downing ND, Forward DP, et al. Surgical treatment of distal radial fractures with a volar locking plate versus conventional percutaneous methods: a randomized controlled trial. J Bone Joint Surg Am 2013;95(19):1737-1744

7 Costa ML, Achten J, Parsons NR, et al; DRAFFT Study Group. Percutaneous fixation with Kirschner wires versus volar locking plate fixation in adults with dorsally displaced fracture of distal radius: randomised controlled trial. BMJ 2014;349. Doi: 10.1136/bmj.g4807

8 Beck JD, Harness NG, Spencer HT. Volar plate fixation failure for volar shearing distal radius fractures with small lunate facet fragments. J Hand Surg Am 2014;39(4):670-678

9 Lozano-Calderón SA, Doornberg J, Ring D. Fractures of the dorsal articular margin of the distal part of the radius with dorsal radiocarpal subluxation. J Bone Joint Surg Am 2006;88(7):1486-1493

10 Twigt B, Bemelman M, Lansink K, Leenen L. Type C distal radial fractures treated with conventional AO plates: an easy and costsaving solution in a locking plate era. Int Orthop 2013;37(3): 483-488

11 Braziulis K, Rimdeika R, Kregždytè R, Tarasevičius S. Associations between the fracture type and functional outcomes after distal radial fractures treated with a volar locking plate. Medicina (Kaunas) 2013;49(9):399-402

12 Swart E, Nellans K, Rosenwasser M. The effects of pain, supination, and grip strength on patient-rated disability after operatively treated distal radius fractures. J Hand Surg Am 2012;37(5): 957-962 
13 Arora R, Lutz M, Hennerbichler A, Krappinger D, Espen D, Gabl M. Complications following internal fixation of unstable distal radius fracture with a palmar locking-plate. J Orthop Trauma 2007; 21(5):316-322

14 Souer JS, Ring D, Jupiter J, Matschke S, Audigé L, Marent-Huber M; LCP Distal Radius Study Group. Comparison of intra-articular simple compression and extra-articular distal radial fractures. J Bone Joint Surg Am 2011;93(22):2093-2099

15 Soong M, Earp BE, Bishop G, Leung A, Blazar P. Volar locking plate implant prominence and flexor tendon rupture. J Bone Joint Surg Am 2011;93(4):328-335

16 Cross AW, Schmidt CC. Flexor tendon injuries following locked volar plating of distal radius fractures. J Hand Surg Am 2008; 33(2):164-167

17 Lutz K, Yeoh KM, MacDermid JC, Symonette C, Grewal R. Complications associated with operative versus nonsurgical treat- ment of distal radius fractures in patients aged 65 years and older. J Hand Surg Am 2014;39(7):1280-1286

18 Drobetz H, Kutscha-Lissberg E. Osteosynthesis of distal radius fractures with a volar locking screw plate. Int Ortho 2003;27:1-6

19 Dumont C, Fuchs M, Folwaczny EK, Heuermann C, Stürmer KM. [Results of palmar T-plate osteosynthesis in unstable fractures of the distal radius] [in German]. Chirurg 2003;74(9):827-833

20 Krimmer H, Pessenlehner C, Hasselbacher K, Meier M, Roth F, Meier R. [Palmar fixed angle plating systems for instable distal radius fractures] [in German]. Unfallchirurg 2004;107(6):460-467

21 Orbay JL, Fernandez DL. Volar fixed-angle plate fixation for unstable distal radius fractures in the elderly patient. J Hand Surg Am 2004;29(1):96-102

22 Wei J, Yang TB, Luo W, Qin JB, Kong FJ. Complications following dorsal versus volar plate fixation of distal radius fracture: a metaanalysis. J Int Med Res 2013;41(2):265-275 